\title{
Symptom cluster and severity among women with breast cancer undergoing chemotherapy in Saudi Arabia
}

\author{
Alwah Mohammed Alkathiri and Ghada Khalid Albothi \\ King Saud University, Saudi Arabia \\ Corresponding author's e-mail: nurse-ambitious@hotmail.com
}

\begin{abstract}
The aim of this descriptive cross-sectional longitudinal study was to identify symptom clusters present in women with breast cancer undergoing chemotherapy treatment in Saudi Arabia using different symptom dimensions (i.e., frequency, severity, distress). A sample of 100 women with breast cancer undergoing chemotherapy was conducted. Symptoms were assessed using the symptom experience scale. Two symptom clusters were identified and stayed approximately constant across different symptom dimensions. The first cluster consisted of nausea, loss of appetite, and sleep disturbance. The second cluster consisted of pain, fatigue, bowel pattern, concentration, appearance, and sleep disturbance. However, the symptom clusters seemed to be dynamic over time. Future research should further investigate symptom clusters trajectories over time.
\end{abstract}

Keywords: Breast cancer, clusters symptom, chemotherapy, frequency, severity.

\section{INTRODUCTION}

Breast cancer is not only a significant problem in Saudi Arabia but is also considered tobe one of the most common causes of cancer-related mortality worldwide. Thousandsof women throughout the world have been clinically diagnosed with breast cancer (Alghamdiet al,2013;American cancer society, 2013).Despite the relatively low incidence of breast cancerin Saudi Arabia compared to other countries, it has been the most common cancer among Saudi females for thepast 12 consecutive years (Alghamdiet al,2013,El Saghir et al, 2007). Data on female patients with invasive breast carcinoma reported from different regions in Saudi Arabia show that most patients are in the age group of 40 to 50 years and were predominantly premenopausal. More than $50 \%$ were stage II and III, while ductal carcinoma in situ represented $<5 \%$ of this population (Ibrahim et.al, 2008).

Breast cancer and its treatment lead to multiple symptoms. Knowledge of these symptoms is important. Breast cancer treatment-related symptoms vary according to the type of treatment (Honea et al,2007). The most common CTX side effects include fatigue, depression, sleep problems, pain, nausea, vomiting, mucositis, anxiety, loss of concentration, andproblems with memory (Rinder, 2005). The most common RT side effects include fatigue, sleep problems, pain, difficulty oncontracting, skin problems, and itching (Kimet al,2008). Themost obvious side effects of HT include sleep disturbance, mood changes, and fatigue. Finally,common BT side effects include fatigue, allergic reactions, fever, rash, headaches, andarthralgias (Polovichet al,2005).

The concept of symptom clusters was first used in psychology and psychiatry as a basis for disease classification and diagnosis of psychiatric disorders, and then, in general medicine to investigate symptom associations and the underlying pathophysiology of diseases (Kimet al,2005). In oncology nursing research, the idea of clustering symptoms was first mentioned by Sarna and Brecht (1997), who clustered symptoms using factor analysis to examine symptom distress among lung cancer patients.

There is scarcity of studies about symptom clustering among cancer patients in Arabic countries as well as 
Saudi Arabia. Instead the researcher will review the literature worldwide. One study identified symptom clusters among women with breast cancer undergoing HT (Glaus et al, 2006). The researchers explored how frequently menopausal symptoms occurred and how symptoms clustered in 375 women. Most of the women were post-menopausal, had early breast cancer (81\%), and were taking tamoxifen $(72 \%)$. A specific scale to assess side effects of HT in women with breast cancer was used. Symptoms were clustered by occurrence using cluster analysis. One symptom cluster was found and included five symptoms: hot flashes, tiredness, vaginal dryness, weight gain, and decreased sexual interest.

Suwisithet al (2010) identified symptom clusters in 320 Thai women undergoing CTX for breast cancer in four outpatient cancer clinics. Most of the women had second $(51.6 \%)$ or third $(27.5 \%)$ stage breast cancer and were newly diagnosed $(73.4 \%)$. They were young with a mean age of 47.3 years ( $S D=8.8$ years). Twenty-five symptoms from the MSAS were included in the analysis. Using the severity and distress dimensions, symptoms were clustered using the FA approach. In the symptom severity dimension, four symptom clusters were found: emotional, GI and fatigue, image-related cutaneous symptoms, and pain and discomfort. In the symptom distress dimension, three symptom clusters were found: emotional and pain, $\mathrm{Gl}$ and fatigue, and image-related cutaneous symptoms. There were many similarities in the two groups of symptom clusters. The symptoms that were clustered by severity explained more of the variance in the functional status $(19.8 \%)$ than symptoms clustered by distress $(17.4 \%)$.

In the study by So et al (2009), 215 Chinese women with breast cancer were examined for the symptom cluster of fatigue, pain, anxiety, and depression. Most of the patients had second $(52 \%)$ or third $(32 \%)$ degree breast cancer and were receiving CTX $(60 \%)$ or RT $(40 \%)$. The symptoms were measured by severity dimension by three symptom specific scales (Brief Fatigue Inventory [BFI], Brief Pain Inventory [BPI], Hospital Anxiety and Depression Scale [HADS]). The results showed significant correlations among the symptoms ranging from 0.25 (pain-depression) to 0.63 (anxiety-depression). These correlations supported the existence of the symptom cluster.

In a sample of 104 patients, breast cancer was the most common type of cancer and represented $80.6 \%$ of the sample. Three types of CTX were included: anthracyclines $(78.7 \%)$, taxanes $(2.9 \%)$, and platinumbased $(18.5 \%)$. Symptoms were assessed using the MSAS at three time points: the day of the first cycle of CTX (T1), end of cycle 1 (T2), and the end of cycle 2 (T3). The RFA was used to determine available symptom clusters based on severity and occurrence approaches. In the severity approach, nausea clustered with pain and lack of energy at T2 and lack of energy and feeling bloated at T3. In the occurrence approach, nausea clustered with pain, taste change, lack of energy, dizziness, appetite loss, and vomiting at T2 and with pain and feeling bloated at T3 (Molassiotis et al., 2012).

The overall purpose of this descriptive study is to explore the presence and characteristics of symptom clusters among Saudi women undergoing intravenous CTX for breast cancer. This study addresses existing gaps in published research literature on symptomclusters among Saudi women with breast cancer undergoing CTX. It helps to identify more preciselycommon symptom clusters and their components in this population.A better understanding of this relationship may lead to the discovery of new innovations insymptom management, development of more targeted intervention strategies. In addition, it may increase economicbenefits and improve health outcomes such as QOL and functional status.

\section{MATERIALS AND METHODS}

Eligible patients (114) were recruited from two university affiliated hospitals and three cancer centers in Riyadh and Jeddah between May-December, 2014. They were stratified by site and randomized at each site based on their sleeping history (good/poor) and the number of CTX cycles prescribed (four or more than four). To be eligible, participants had to be (a) women 19 years and older; (b) initial diagnosis of stages I to IIIA breast cancer; (c)postmodified radical mastectomy or lumpectomy; and (d) scheduled to begin four anthracyclinebased(A/C) intravenous CTX treatments with or without four additional taxane treatments; . Exclusion criteria included self reported history of diagnosis of co-morbidities associated with poor sleep and fatigue (includingchronic insomnia, chronic fatigue syndrome, unstable heart, lung or neuromuscular disease,insulin-dependent diabetes, sleep apnea, chronic oral steroid therapy, and nightshiftemployment).Of the women screened, 14 were excluded from the study; 9 refused to participatebecause they were not interested, and 5 did not meet the inclusion criteria.

Symptom clustering was assessed using Symptom Experience Scale (SES) (Saramel et al., 1996). In addition, baseline questionnaires were used to gather demographic information about participants. The SES consists of 24 items, rated on a five-point Likert scale that ranges from 0 (most positive result) to4 (most negative result). Each point in the scale is connected with descriptive words to facilitateunderstanding. The descriptors allow for total absence of the symptom in all three symptomdimensions. The SES is administered as a 3-page self-report questionnaire and takesapproximately 15 minutes to complete. It assesses symptoms present during the past week. Toobtain the total symptom experience score, all items are summed. Scores can range from 0 to 96 . The SES is valid and reliable for measuring symptom experience in oncology patients 
Table1. Demographic characteristics of the sample

\begin{tabular}{llc}
\hline Characteristic & Mean (SD) & Range \\
\hline Age (years) & $49(10)$ & $35-66$ \\
Working Hours & $35(19)$ & $0-65$ \\
\hline & Categories & $\boldsymbol{N}(\%)$ \\
\hline \multirow{2}{*}{ Education } & Up to High School & $34(34)$ \\
& Some College or more & $66(66)$ \\
Marital Status & Married & $88(88)$ \\
& Non-Married & $12(12)$ \\
Employment & Employed & $55(55)$ \\
Household Income (SR) & Non-Employed & $45(45)$ \\
(year) & Less than 100,000 & $48(48)$ \\
Nationality & Over 100,000 & $52(52)$ \\
& Saudi & $89(89)$ \\
& Non- Saudi & $11(11)$ \\
\hline
\end{tabular}

(Saramel et al., 1996). Content and construct validity were used to evaluate the scale. Content validity was confirmed by a panel of expert oncologists. Construct validity was tested by comparing total symptom experience scores between women receiving CTX $(\mathrm{n}=$ 74) and women who were not receiving any adjuvant treatment $(n=143)$. Scores were significantly higher for women receiving CTX. Internal consistency reliability was estimated with Cronbach's alpha and was 0.94 for the total scale (Saramel et al., 1996).

The model of Brislin et al (1973) was usedto translate of the SES into Arabic (with permission from the author). Translation sought to obtain conceptual equivalence between the original English version and a culturally acceptable Arabic version. Pretesting of the Arabic language tool was done with 10 women with breast cancer; they found the tool easy to complete.Internal consistency reliability for the Arabic version was estimated with Cronbach's alpha and was 0.86 for the total scale.

Women who had undergone surgery for breast cancer and scheduled an appointment toreceive their first CTX were introduced to the study by the clinic nurse who briefly explained the study to them and asked if they would be willing to speak to the research nurse. The research nurse contacted potential participants, to further explain the study and check for eligibility criteria. If the woman met the inclusion criteria and agreed to participate in the study, she was given an informed consent to sign, and was then given a baseline questionnaire to complete before beginning her initial treatment. The SES asks about symptoms experienced in the week of the scale administration and was administered one week after each CTX. Personal, health and illness, and treatment characteristics were measured at the beginning of the study, before starting the treatment. Data were analyzed using SPSS for Windows software version 17.0.1 (SPSS, Inc., Chicago, IL, USA). Descriptive statistics were used to describe the demographics, clinical characteristics, and symptom dimensions, as well as to assess study variables for any violation of statistical assumptions. Descriptive statistics included the mean, standard deviation (SD), and range for continuous variables and frequencies and percentages for categorical variables. A pvalue $\leq 0.05$ was considered statistically significant. All tests were twotailed.The study protocol was approved by theethics committee of each study's sites. Writteninformed consent was obtained from all participants.

\section{RESULTS}

The demographic characteristics of the sample are presented in Table 1. All participants in the study were female with breast cancer $(n=100)$. The mean age of the women was 49 years $(S D=10)$ and ranged from 35 to 66 . The majority of the women were Saudi $(89 \%)$, married $(88 \%)$, and had at least a college education $(66 \%)$. In addition, most were employed (55\%), worked on average 35 hours per week $(S D=19)$, and had a household income of over SR 100,000 per year (52\%).

The clinical characteristics of the sample are summarized in Table 2. Thirty-three percent of the women had first stage breast cancer and $67 \%$ had second or third stage. The majority of the women had breast cancer with positive estrogen receptors (75\%) and progesterone receptors (66\%). Approximately half of the women (52\%) had no lymph node involvement. All women had surgery for breast cancer approximately three to four weeks prior to beginning of CTX; $56 \%$ underwent modified mastectomy and $43 \%$ underwent lumpectomy. The mean BMI was $28.7(\mathrm{SD}=6.1)$ and ranged from 16 to 53 . 
Table 2 Clinical Characteristics of the Sample

\begin{tabular}{lcc}
\hline Characteristic & Mean (SD) & Range \\
\hline BMI* $^{*}$ & $28.7(6.1)$ & $16-53$ \\
Hemoalobin level (baseline) & $13.1(1.2)$ & $10.3-16.4$ \\
Surgical Procedure & Categories & $N(\%)$ \\
Cancer Stage & Lumpectomy & $95(416)$ \\
& Modified Mastectomv & $123(56.4)$ \\
Lymph Node Status & I & $72(33.2)$ \\
& Positive, I to 3 & $145(66.8)$ \\
Estrogen Receptor Status & Positive, 4 to 9 & $26(11.9)$ \\
& Negative & $115(52.5)$ \\
Progesterone Receptor Status & Positive & $160(75.5)$ \\
& Neqative & $52(24.5)$ \\
& Positive & $106(66.2)$ \\
\hline
\end{tabular}

*BMI: Body Mass Index

Table 3 Means and Standard Deviations for Symptoms at the Cycle Three of CTX $(n=100)$

\begin{tabular}{llcc}
\hline \multirow{2}{*}{ Symptoms } & \multicolumn{3}{c}{ Symptom Dimensions } \\
\cline { 2 - 4 } & $\begin{array}{c}\text { Frequency } \\
\text { Mean (SD) }\end{array}$ & $\begin{array}{c}\text { Severity } \\
\text { Mean (SD) }\end{array}$ & $\begin{array}{c}\text { Distress } \\
\text { Mean (SD) }\end{array}$ \\
\hline Nausea $^{*}$ & $1.35(1.04)$ & $1.30(0.94)$ & $1.35(0.89)$ \\
Pain $^{*}$ & $0.80(0.82)$ & $0.85(0.87)$ & $0.95(0.92)$ \\
Appetite* $^{*}$ & $1.49(1.04)$ & $1.26(0.94)$ & $1.20(0.78)$ \\
Sleep Disturbance $^{*}$ & $1.41(1.01)$ & $1.41(0.95)$ & $1.36(0.83)$ \\
Fatigue $^{*}$ & $2.18(0.99)$ & $1.85(0.76)$ & $1.57(0.70)$ \\
Bowel Pattern $^{*}$ & $1.41(1.10)$ & $1.24(0.92)$ & $1.35(0.94)$ \\
Concentration $^{*}$ & $1.07(0.88)$ & $1.03(0.84)$ & $1.10(0.85)$ \\
Appearance $^{*}$ & $0.94(1.05)$ & $0.78(0.88)$ & $0.98(1.00)$ \\
\hline
\end{tabular}

* The scores range from 0 (most positive result) to 4 (most negative result)

The symptom frequency, severity, and distress mean scores for the symptoms are summarized in table 3 . The women who did not have symptoms were still included in calculating mean symptom severity and distress scores. All symptoms occurred in more than $20 \%$ of the women and were included in further analysis. However, most of the symptoms occurred with low frequency. The mean symptom frequency scores for the SES ranged from 0.80 for pain to 2.18 for fatigue on a scale of 0 to 4 .

The overall score for symptom distress was relatively mild-to-moderate. The mean symptom distress score for the SES symptoms ranged from 0.95 for pain to 1.57 for fatigue on a scale of 0 to 4 . The four symptoms that caused the most distress from the SES were fatigue $(1.57 \pm 0.70)$, sleep disturbance $(1.36 \pm 0.83)$, nausea $(1.35 \pm 0.89)$, and bowel pattern $(1.35 \pm 0.94)$. The overall score for symptom severity was relatively mild-tomoderate. The mean symptom severity score for the SES symptoms ranged from 0.78 for appearance to 1.85 for fatigue on a scale of 0 to 4 . The four most severe symptoms from the SES were fatigue $(1.85 \pm 0.76)$, sleep disturbance $(1.41 \pm 0.95)$, nausea $(1.30 \pm 0.94)$, and appetite $(1.26 \pm 0.94)$. It is important to note that scores for the three different symptom dimensions were similar. For example, women who had severe symptoms also had more symptom distress.

The symptom prevalence (\% of the sample) and severity (mean + standard deviation) across the four time points are presented in Table 4. The most prevalent symptom was fatigue, which ranged from $89.2 \%$ to $98.3 \%$, followed by sleep disturbance, pain, and concentration problems all with prevalence above $50 \%$ at each time point. The least prevalent symptoms were anxiety and depression, rated fewer than $50 \%$ at all time points. In general, symptoms were more prevalent during CTX (T2 and T3). However, pain was more prevalent before and after CTX. During CTX, all symptoms had prevalence greater than $20 \%$ and therefore were included in further analysis. The mean symptom severity scores for the SES symptoms ranged from 0.71 for appearance to 1.90 for fatigue. Six symptoms, namely nausea, appetite, sleep disturbance, fatigue, bowel pattern, and 
Table 4 Symptom Prevalence and Severity across Time Points $(n=100)$

\begin{tabular}{|c|c|c|c|c|c|c|c|c|}
\hline \multirow[b]{2}{*}{ Symptoms } & \multicolumn{2}{|c|}{ T1 } & \multicolumn{2}{|c|}{ T2 } & \multicolumn{2}{|c|}{ T3 } & \multicolumn{2}{|c|}{$\mathrm{T} 4$} \\
\hline & $\begin{array}{c}\text { Prevalence } \\
\%\end{array}$ & $\begin{array}{l}\text { Severity } \\
\text { Mean (SD) }\end{array}$ & $\begin{array}{c}\text { Prevalence } \\
\%\end{array}$ & $\begin{array}{c}\text { Severity } \\
\text { Mean (SD) }\end{array}$ & $\underset{\%}{\text { Prevalence }}$ & $\begin{array}{l}\text { Severity } \\
\text { Mean (SD) }\end{array}$ & $\begin{array}{c}\text { Prevalence } \\
\%\end{array}$ & $\begin{array}{c}\text { Severity } \\
\text { Mean (SD) }\end{array}$ \\
\hline Nausea' & 22.1 & $0.32(0.68)$ & 79.1 & $1.30(0.94)$ & 80.4 & $1.32(0.95)$ & 13.7 & $0.16(0.44)$ \\
\hline Pain" & 84.8 & $1.28(0.79)$ & 58.8 & $0.85(0.87)$ & 59.8 & $0.86(0.89)$ & 61.0 & $0.88(0.86)$ \\
\hline Appetite' & 41.2 & $0.45(0.62)$ & 82.9 & $1.26(0.94)$ & 82.1 & $1.25(0.93)$ & 28.6 & $0.34(0.63)$ \\
\hline Sleep & 76.0 & $1.25(0.91)$ & 84.0 & $1.41(0.95)$ & 80.0 & $1.28(0.95)$ & 64.8 & $0.90(0.90)$ \\
\hline Fatigue & 89.2 & $1.21(0.64)$ & 97.9 & $1.85(0.76)$ & 98.3 & $1.90(0.81)$ & 94.5 & $1.32(0.64)$ \\
\hline Bowel Patten* & 37.7 & $0.46(0.69)$ & 80.2 & $1.24(0.92)$ & 77.8 & $1.18(0.88)$ & 29.1 & $0.37(0.64)$ \\
\hline Concentration & 54.7 & $0.66(0.69)$ & 73.1 & $1.03(0.84)$ & 72.2 & $1.08(0.91)$ & 59.9 & $0.74(0.71)$ \\
\hline Appearance" & 25.5 & $0.26(0.49)$ & 57.0 & $0.78(0.88)$ & 52.8 & $0.71(0.84)$ & 33.5 & $0.42(0.70)$ \\
\hline
\end{tabular}

*The scores severity range from 0 (most positive result) to 4 (most negative result),

concentration, had mean symptom severity scores greater than one during both CTX cycles. However, no symptom exceeded a mean severity score of two on the 0 to 4 scale. Pain, which had a mean severity score less than one during CTX, was the most severe symptom reported at T1. In addition, fatigue was the only symptom from the SES with a severity greater than one across all time points.

Two symptom clusters were identified: the GI symptom cluster and the treatment-related symptom cluster. The GI symptom cluster included two symptoms (nausea and appetite) when measured by severity dimension, and three symptoms (nausea, appetite, and sleep disturbance) when measured by other dimensions. The treatment-related symptom cluster included five symptoms (pain, fatigue, concentration, bowel pattern, and appearance) when measured by frequency and distress dimensions (pain, fatigue, concentration, bowel pattern, appearance, and sleep disturbance) when measured by severity dimension. The symptom clusters were able to explain from $35.22 \%$ to $39.65 \%$ of the total variance. The symptom clusters were stable with Cronbach alpha coefficients ranging from 0.62 to 0.80 . The correlations between the two symptom clusters were moderate and ranged from 0.64 to .66 . Two symptoms fatigue and sleep disturbance, loaded strongly on both clusters, indicating they were related to both clusters.

\section{DISCUSSION}

To my knowledge, this is the first study done to date on symptom clusters among women with breast cancer undergoing CTX in Saudi Arabia. The biggest strength of the study was its homogeneous sample; all women had early stage breast cancer and were having only CTX treatment.

During treatment, all symptoms occurred in more than $20 \%$ of the women. However, most symptoms occurred occasionally and had mild-to-moderate severity. Similar results were found in other studies (Kim et al., 2006;
Suwisith et al., 2010). Suwisith et al. reported that most symptoms occurred occasionally, and mean severity scores ranged from 1.32 to 2.66 on a $1-4$ scale. Furthermore, studies that explored subgroups in sickness behavior and Gl symptom clusters (Dodd et al., 2010; Given et al., 2001; Golan-Vered\&Pud, 2012; Kim et al., 2012; Miaskowski et al., 2006; Pud et al., 2008) reported that $19 \%$ to $62.5 \%$ of patients were in the low symptom subgroup, which indicates that most patients experienced low symptom severity.

The causes of low symptom severity in this study may be related to some inclusion criteria (i.e., early breast cancer) or the method by which the mean score was calculated (including all patients regardless of whether they had a symptom or not). In addition, patients with more severe symptoms may have been less likely to enroll in the study or more likely to drop out from the study. One interesting result is that pain had its highest frequency and severity at the baseline. This result was supported by Kim (2006). The high frequency and severity of pain at baseline may be due to surgical procedures that the women underwent one month prior to beginning CTX.

Two symptom clusters were found when clustering symptoms by different dimensions. The GI symptom cluster consisted of nausea and appetite when clustered by severity dimension, and nausea, appetite, and sleep disturbance when clustered by frequency and distress dimensions.

The Gl symptom cluster is common and specific to CTX treatment. All studies that included women with CTX treatment and used all-possible approach have found this cluster. The cluster was less common in the studies that included only RT treatment. Only Matthews et al. (2011) reported a Gl symptom cluster in a sample specific to RT, when they clustered symptoms according to the distress dimension.

Symptoms included in the GI symptom cluster widely vary among the studies. Most studies included nausea in the cluster. Other common symptoms found in the cluster were vomiting, loss of appetite, lack of energy, and 
feeling bloated. Less common symptoms found were dizziness, feeling drowsy, shortness of breath, pain, and bowel patterns. This is the first study that included sleep disturbance in the Gl symptom cluster and it is not clear why sleep disturbance clustered with the GI symptoms when clustering symptoms using frequency and distress dimensions. However, it is important to note that sleep disturbance loaded on both clusters with higher loading on $\mathrm{Gl}$ cluster when clustering by frequency and distress dimension. Sleep disturbance item-total correlations were strong, ranging from 0.39 to 0.43 . In addition, Cronbach's alpha ranged from 0.65 to 0.70 for the GI symptom cluster that included sleep disturbance, and was 0.62 for the cluster with nausea and loss of appetite alone.

The second symptom cluster that was found in the current study was the treatment-related symptom cluster. This cluster consisted of six symptoms when clustered by the severity dimension (pain, fatigue, bowel pattern, concentration, appearance, sleep disturbance) and five symptoms when clustered by frequency or distress dimensions (pain, fatigue, bowel pattern, concentration, appearance). The treatment-related symptom cluster had different names in the literature such as sickness behavior or psycho-neurological symptom cluster. It is a common cluster found in almost all studies that explored symptom clusters during treatment. The number of symptoms in the cluster differs among the studies. In studies that used common symptom approach the cluster consisted of three to four symptoms, most commonly pain, fatigue, and insomnia. In studies that used all possible symptom approach the cluster consisted of three to six symptoms.

Two studies (Kim et al., 2008; So et al., 2009) included depression in the treatment related symptom cluster. In other studies, emotional symptoms (e.g., depression, worrying, feeling irritable, feeling nervous) were clustered alone or with cognitive symptoms (e.g., concentration). One study (Kim et al., 2008) supported clustering of pain, fatigue, and insomnia with emotional and cognitive symptoms such as in the current study. Conversely, in the Suwisith et al. (2010) study, the three key symptoms were in different clusters; insomnia was a part of emotional cluster, fatigue was a part of GI cluster, and pain was a part of pain cluster that consisted of pain, numbness, and dry mouth. It is unclear why the same symptoms clustered differently among the studies. Some reasons may include: 1) using different symptom dimensions when clustering the symptoms, 2) including heterogeneous samples, 3 ) different number and types of symptoms among the studies, or 4) using different methods to extract the number of factors.

It is unclear clustering in which dimension is more comprehensive and beneficial. In the current study there were minimal differences between symptom clusters when clustered by the three symptom dimensions. In the literature, three studies (Kim et al., 2009c; Molassiotis et al., 2012; Suwisith et al., 2010) compared symptoms clustered by different dimensions and found mild-tomoderate differences, and it is not clear which dimension is better to use. According to Kim et al. (2009c), severity dimension fits the data better. However, in the current study, clustering by distress dimension had better reliability and explained more variance.

In conclusion, this study aimed to identify common symptom clusters in women with breast cancer undergoing CTX. Two symptom clusters ( $\mathrm{Gl}$ and treatment-related) were found. However, symptoms in each cluster differed according to the assessment time. The symptom clusters stayed fairly constant when clustering by different symptom dimensions; this indicates that using any dimension should result in consistent, accurate and comprehensive results. Although many findings from this study are preliminary, the findings have implications for further research and clinical practice. The findings will contribute to the comprehensive assessment, prevention, and management of symptoms involved in the common symptom clusters. Furthermore, the findings can encourage researchers to pursue longitudinal studies when trying to better understand symptom clusters.

\section{REFERENCES}

Alghamdi I, Hussain I, Alghamdi M, El-Sheemy M (2013). The incidence rate of female breast cancer in Saudi Arabia: an observational descriptive epidemiological analysis of data from Saudi Cancer Registry 2001-2008. Breast Cancer: Targets and Therapy 2013:5, 103-109.

American Cancer Society (2013). Cancer facts \& figures 2013. Atlanta: American Cancer Society. Retrieved fromhttp://www.cancer.org/acs/groups/content/@epidemiologysurvei lance/documents/document/acspc-036845.pdf

Brislin RW, Lonner WJ, Thorndike RM (1973). Cross-Cultural Research Methods. New York: John Wiley \& Sons, CA: Sage Publications.

Dodd M, Cho M, Cooper B, Miaskowski C (2010). The effect of symptom clusters onfunctional status and quality of life in women with breast cancer. European Journal ofOncology Nursing, 14, 101110. doi: 10.1016/j.ejon.2009.09.005

El Saghir NS, Khalil MK, Eid T (2007). Trends in epidemiology and management of breast cancer in developing Arab countries: a literature and registry analysis. Int J Surg. ;5(4):225-233

Given B, Given C, Azzouz F, Stommel M (2001). Physical functioning of elderly cancer

patients prior to diagnosis and following initial treatment. Nursing Research, 50, 222-232. http://dx.doi.org/10.1097/00006199200107000-00006

Glaus A, Boehme C, Thürlimann B, Ruhstaller T, Hsu Schmitz S, Morant R, Von Moos R (2006). Fatigue and menopausal symptoms in women with breast cancer undergoing hormonal cancer treatment. Annals of Oncology, 17, 801-806. http://dx.doi.org/10.1093/annonc/mdl030http://dx.doi.org/10.1097/N CC.0b013e3182277222

Honea N, Brant J, Beck S (2007). Treatment-related symptom clusters. Seminars in Oncology Nursing, 23, 142-151. http://dx.doi.org/10.1016/j.soncn.2007.01.002

Ibrahim EM, Zeeneldin AM, Sadiq BB, Ezzat AA (2008). The present and the future of breast cancer burden in the Kingdom of Saudi Arabia. Med Oncol .25:387-93.

Kim HJ, (2006) Treatment-related symptom clusters in breast cancer: A secondary analysis(Doctoral dissertation). Retrieved from ProQuest Dissertations and Theses Database. (AAT 3225481).

Kim H, McGuire D, Tulman L, Barsevick A (2005). Symptom clusters: Concept analysis 
and clinical implications for cancer nursing. Cancer Nursing, 28, 270282. http://dx.doi.org/10.1097/00002820-200507000-00005

Kim H, Barsevick A, Tulman L, McDermott P (2008). Treatment-related symptom clusters in breast cancer: A secondary analysis. Journal of Pain and Symptom Management, 36, 468-479. http://dx.doi.org/10.1016/j.jpainsymman.2007.11.011

Kim E, Jahan T, Aouizerat B, Dodd M, Cooper B, Paul S, Wara W (2009). Differences in symptom clusters identified using occurrence rates versus symptom severity ratings in patients at the end of radiation therapy. Cancer Nursing, 32, 429-436. doi: 10.1097/NCC.0b013e3181b046ad

Kim HJ, Barsevick AM, Beck SL, Dudley W (2012). Clinical subgroups of a psychoneurologic symptom cluster in women receiving treatment for breast cancer: A secondary analysis. Oncology Nursing Forum, 39, E20-E30. doi: 10.1188/12.ONF.E20- E30

Matthews E, Schmiege S, Cook P, Sousa K (2011). Breast cancer and symptom clusters

during radiotherapy. Cancer Nursing, 0, 1-11.

Miaskowski C, Cooper B, Paul S, Dodd M, Lee K, Aouizerat B, Bank A (2006).Subgroups of patients with cancer with different symptom experiences and quality-of-lifeoutcomes: A cluster analysis. Oncology Nursing Forum, 33, E79E89.http://dx.doi.org/10.1188/06.ONF.E79-E89

Molassiotis A, Farrell C, Bourne K, Brearley SG, Pilling M (2012). An exploratory study to clarify the cluster of symptoms predictive of chemotherapy-related nausea using random forest modeling.

Journal of Pain and Symptom Management, 44, 692-703. http://dx.doi.org/10.1016/j.jpainsymman.2011.11.003

Polovich M, White J, Kelleher L (Ed 2.). (2005). Chemotherapy and Biotherapy Guidelines and Recommendations for Practice. Pittsburgh, PA: Oncology Nursing Society

Pud D, Ben Ami S, Cooper BA, Aouizerat BE, Cohen D, Radiano R, Kachta $\mathrm{O}(2008)$. The symptom experience of oncology outpatients has a different impact onquality-of-life outcomes. Journal of Pain and Symptom Management, 35, 162170.http://dx.doi.org/10.1016/j.jpainsymman.2007.03.010

Ridner S (2005). Quality of life and a symptom cluster associated with breast cancer treatmentrelated lymphedema. Supportive Care in Cancer, 13, 904-911. http://dx.doi.org/10.1007/s00520-005-0810-y
Samarel N, Leddy SK, Greco K, Cooley ME, Torres SC, Tulman L, Fawcett J (1996). Development and testing of the symptom experience scale. Journal of Pain andSymptom Management, 12, 221-228. Retrieved fromhttp://www.journals.elsevier.com/journal-ofpain-and-symptom-management/

Sarna L, Brecht ML (1997). Dimensions of symptom distress in women with advanced lung cancer: A factor analysis. Heart \& Lung: The Journal of Acute and Critical Care, 26, 23-30. http://dx.doi.org/10.1016/S0147-9563(97)90006-6

So W, Marsh G, Ling W, Leung F, Lo J, Yeung M, Le G (2009). The symptom cluster of fatigue, pain, anxiety, and depression and the effect on the quality of life of women receiving treatment for breast cancer: A multicenter study. Oncology Nursing Forum, 36, E205E214. http://dx.doi.org/10.1188/09.ONF.E205-E214

Suwisith N, Hanucharurnkul S, Dodd M, Vorapongsathorn $T$, Pongthavorakamol K, Asavametha N (2010). Symptom clusters and functional status of women with breast cancer. Thai Journal of Nursing Research, 12, 153-165. Retrieved from http://thailand.digitaljournals.org/index.php/TJNR 